\title{
Risk Stratification Of Diffuse Large B-Cell Lymphoma With Interim PET/CT By Combining Deauville Scores And International Prognostic Index
}

This article was published in the following Dove Press journal: Cancer Management and Research

\author{
Hongwei Sun ${ }^{1,2, *}$ \\ Zhan $\mathrm{Yu}^{3, *}$ \\ Ning $\mathrm{Ma}^{3}$ \\ Jie Zhou ${ }^{3}$ \\ Rongrong $\operatorname{Tian}^{3}$ \\ Ming Zhao ${ }^{3}$ \\ Tong Wang (D) \\ 'Department of Health Statistics, School \\ of Public Health, Shanxi Medical \\ University, Taiyuan City, Shanxi 03000I, \\ People's Republic of China; ${ }^{2}$ Department \\ of Health Statistics, School of Public \\ Health And Management, Binzhou \\ Medical University, Yantai City, Shandong \\ 264003, People's Republic of China; \\ ${ }^{3}$ Department of PET/CT Center, The \\ Tumor Hospital of Shanxi Province, \\ Taiyuan City, Shanxi 030000, People's \\ Republic of China \\ *These authors contributed equally to \\ this work
}

Correspondence: Tong Wang

Department Of Health Statistics, School Of Public Health, Shanxi Medical

University, Xinjiannanlu 56, Taiyuan City,

Shanxi 03000I, People's Republic of China

$\mathrm{Tel} / \mathrm{Fax}+86-35 \mathrm{I}-4 \mathrm{I} 35397$

Email tongwang@sxmu.edu.cn

Ming Zhao

Department Of PET/CT Center, The

Tumor Hospital Of Shanxi Province,

Zhigongxin Street 3, Taiyuan City, Shanxi

030013 , People's Republic of China

$\mathrm{Tel} / \mathrm{Fax}+86-35 \mathrm{I}-488 \mathrm{I} 6 \mathrm{II}$

Email zm19701025@163.com
Purpose: Prognostic evaluation using interim positron emission tomography/computed tomography (interim PET/CT; I-PET) remains controversial. For any predictor, the prognosis of patients around its cutoff value is most uncertain. If the patients around the cutoff value could be subdivided by another factor, like the international prognostic index (IPI), it may improve the predictive power of I-PET. The combination of I-PET and IPI for risk stratification of patients was explored in this study.

Patients and methods: One hundred and eleven diffuse large B-cell lymphoma (DLBCL) patients treated with R-CHOP therapy were included retrospectively, 59 of whom underwent PET/CT after three or four cycles of treatment (I-PET). Fifty-two patients underwent PET/ CT after five or six cycles of treatment (end of treatment; E-PET).

Results: When Deauville 5-point scale (5-DS) scores of 4-5 were classified as a positive scan (denoted by DS [score 4]), there was no significant difference in progression-free survival (PFS) between I-PET positive and negative patients $(P=0.151)$. Further, patients with 5-DS score 3 and high IPI were stratified into I-PET positive-, whereas those with 5-DS score 3 and low IPI were classified into I-PET negative scan groups. Under this stratification, there was a significant difference in PFS between I-PET positive and negative patients $(P=0.001)$. The sensitivity, positive predictive value, and negative predictive value for 2-year PFS for the combination score were higher than DS (score 4 ) alone (66.7\% vs 33.3\%, 50.0\%vs 37.5\%, 93.6\% vs $88.2 \%$ ) whereas specificity was almost the same $(90.0 \%$ vs $88.0 \%)$.

Conclusion: Subdivision of patients with 5-DS score 3 by IPI improved prognostic prediction accuracy. The IPI adds strength to 5-DS in I-PET to detect patients with good or poor prognosis. Compared with other combinations of I-PET and IPI, dividing the patients around the cutoff value of 5-DS by IPI was easily accepted by clinicians and allowed them to decide on further treatment practically.

Keywords: Deauville 5-point scale, interim positron-emission tomography/computed tomography, IPI, cutoff value, subdivision

\section{Introduction}

Diffuse large B-cell lymphoma (DLBCL) is the most common type of aggressive lymphoma. Some patients can be cured after standard treatment, but there remains up to $30-40 \%$ of the patients who suffer relapses that are refractory to first-line immunochemotherapy. ${ }^{1}$ Early identification of such patients is very important in clinical treatment decisions. Treatment continuation or even de-escalation can be performed in patients with good prognosis to reduce toxicity without adversely affecting outcome. 
Treatment intensification or modification can be proposed in those patients with poor prognosis to improve cure rates. ${ }^{2}$

The International Prognostic Index (IPI) based on age, performance status, lactate dehydrogenase (LDH) level, number of extranodal sites, and lymphoma stage is the most widely adopted prognostic tool for patients with DLBCL. However, within each IPI score, patient outcomes are still heterogeneous. ${ }^{3}$

Another method for assessment of the treatment response and prognosis of DLBCL patients is by positron emission tomography-computed tomography (PET/CT) after a few cycles of (immuno) chemotherapy, which is also known as interim FDG-PET (I-PET). ${ }^{4,5}$ Compared with the reported high predictive accuracy of end of treatment PET/CT (E-PET), an early response assessment using I-PET is more controversial due to variable results. ${ }^{2,6}$

The Deauville 5-point scale (5-DS) ${ }^{7}$ is the most widely adopted criteria for both I-PET and E-PET. ${ }^{8}$ Meignan et $\mathrm{al}^{7}$ reported the consensus guideline for 5-DS classification but did not define the cutoff value of 5-DS for classification of positive or negative scans. In previous studies, ${ }^{9-12} 5$-DS of scores of 1-3 were defined as negative scans, and scores of 4-5 were considered to be a positive result in both I-PET and E-PET. Kim et $\mathrm{al}^{8}$ evaluated the ability of I-PET to identify risk using different cutoff values of 5-DS and showed that a cutoff score of 5 at I-PET had an alternative clinical implication for identifying patients at significant risk.

IPI reflects pre-treatment factors relating to prognosis, whereas the I-PET result relates to chemosensitivity. 5-DS criteria were also combined with IPI to improve the predictive accuracy in some studies. Chow et $\mathrm{al}^{13}$ reported I-PET with IPI as a nomogram based on a bootstrapped Cox regression model to improve predictive accuracy. However, the cutoff value of this nomogram was not given, so it is hard for clinicians to discriminate between good- or poor-outcome patients for further treatment options. Nols et $\mathrm{al}^{14}$ reported that combination of the qualitative and quantitative indices of I-PET with IPI was highly predictive of outcome in DLBCL. This type of combination divided patients into three groups, double positive, where two indices are positive; double negative, where two indices are negative; and a group with only one positive index. For patients where only one index was positive, the prognosis (eg, 2-year progression-free survival (PFS) of I-PET- or IPI-positive patients were 60\% and $69 \%$, respectively) was usually between that of the doublepositive (2-year $0 \%$ PFS) and double-negative (2-year $88 \%$ PFS) groups. But Nols et $a^{14}$ did not classify this group of patients as either a positive group with poor prognosis or a negative group with good prognosis; thus, it was still difficult for clinicians to decide on further treatment of these patients.

Therefore, it is clear that these two methods of combining I-PET and IPI were not practical for informing treatment decisions. For any predictor, the prognosis of patients around its cutoff value was most uncertain. Patients with 5-DS scores of 3 showed uptake intensity between the mediastinum and the liver, and were considered to have partially progressed. According to the results of a meta-analysis, ${ }^{6}$ if these patients were classified as positive, then the sensitivity of I-PET for prognosis increased but the specificity decreased. Conversely, if these patients were classified as negative, then sensitivity decreased but specificity increased. This illustrates that patients with 5-DS scores of 3 are heterogeneous, with differing prognoses. Subdividing patients with 5-DS scores of 3 based on other predictive factors may improve predictive accuracy.

In our study, subdivision of patients with 5-DS scores of 3 according to the International Prognostic index (denoted DS+IPI) was considered. DS+IPI combined the features of DS and IPI, resulting in an I-PET negative group consisting of patients with DS scores 1-2 or 3 but low IPI, and an I-PET positive group consisting of patients with DS scores $4-5$ or 3 but high IPI. This combination of I-PET and IPI can classify patients with good or poor prognosis explicitly. The present retrospective study was undertaken to assess the clinical value of DS+IPI in predicting the prognosis of patients with DLBCL.

\section{Materials And Methods}

\section{Patients}

Our investigation was approved by the ethics committee of Shanxi Medical University (Taiyuan, China, ID number: 2018033). Informed consent was not obtained since this was a retrospective study. All personal data were strictly confidential. The study protocol conformed to the ethical guidelines of the Declaration of Helsinki. Our retrospective study included patients with DLBCL visiting the Tumor Hospital of Shanxi Province between April 2015 and April 2017. All patients underwent PET/CT imaging during the treatment or post-treatment period. For each patient, 2 weeks elapsed from the end of the preceding treatment to the scan. Inclusion criteria were as follows: 1) newly diagnosed with DLBCL by histopathological and 
immunohistochemical examination; 2) treated with 6-8 cycles of R-CHOP with a treatment course of 21 days; 3 ) received at least one $18 \mathrm{~F}-\mathrm{FDG} \mathrm{PET} / \mathrm{CT}$ examination; and 4) other malignant tumors or chronic diseases were not identified during the follow-up period. In total, 111 patients were included, of whom 59 underwent PET/CT after 3-4 cycles of treatment (I-PET) and 52 underwent PET/CT after 5-6 cycles of treatment (end of treatment, E-PET). Clinical data obtained from all patients prior to treatment included sex, age, ECOG performance status, number of extranodal sites of involvement, Ann Arbor stage, and serum LDH level, allowing calculation of the IPI.

Clinical follow-up was carried out for all patients once every three months, including histopathological examination and imaging, such as ultrasound. PFS was calculated from the date of diagnosis to first relapse or progression, death of any cause, or last follow-up date. The range of follow-up time was 4-83 months. The median follow-up time was 24 months.

\section{I8F-FDG PET/CT Acquisition}

All patients underwent ${ }^{18} \mathrm{~F}$-fluoro-2-dexoxy-D-glucose $\left({ }^{18}\right.$ F-FDG) PET/CT imaging on a Discovery STE PET/ CT system (GE Healthcare, USA), consisting of a 16detector-row CT scanner. The ${ }^{18}$ F-FDG PET/CT imaging agent was produced by the PET/CT center at the Tumor Hospital of Shanxi Province using ${ }^{18} \mathrm{~F}$ produced with a MINItrace drug synthesis system (GE Healthcare, USA). Eventually, imaging agents were synthesized using a Trace Lab FXN multifunctional composite device. The chemical purity of the drug was greater than $90 \%$.

Patients fasted for at least $4 \mathrm{hrs}$ before intravenous administration of ${ }^{18} \mathrm{~F}-\mathrm{FDG}(5-6 \mathrm{MBq} / \mathrm{kg})$ to ensure a serum glucose level below $11 \mathrm{mmol} / \mathrm{L}$. PET/CT scanning was acquired at $1 \mathrm{hr}$ after 18F-FDG administration. Transmission data were acquired using a low-dose CT scan $(120 \mathrm{kV}, 180 \mathrm{~mA}$, a $512 \times 512$ matrix, $3.75-\mathrm{mm}$ slice thickness), and the scanning range was from vertex to the upper segment of the femur. PET images were acquired by the $3 \mathrm{D}$ model, and the scanning range was the same as for CT. Six to eight bed-boards were collected according to the different heights of patients, with one bed-scanning taking 3 mins. The images were reconstructed using a conventional iterative algorithm (OSEM). Multiplanar reformed PET and CT images were analyzed frame by frame on a Xeleris workstation (GE Healthcare, USA).

\section{PET Scan Interpretation}

$\mathrm{PET} / \mathrm{CT}$ scans were read by two trained imaging physicians. If there was a dispute regarding any given image, it was read by the director and agreement was reached after discussion. Visual analysis of the image was adopted in this study. 5-DS criteria in the National Comprehensive Cancer Network clinical guidelines, 2016, ${ }^{15}$ were used to assess treatment response qualitatively as follows: score 1 , no residual disease; score 2, uptake less than or equal to the mediastinal blood pool; score 3, uptake more than the mediastinal blood pool but less than or equal to the liver uptake; score 4, uptake was moderately greater than that of the liver; score 5, uptake markedly increased compared with the liver or new lesions were detected. Clinically, it is necessary to stratify patients who performed to I-PET into negative scan or positive scan, which indicates a good or bad outcome for informing treatment decisions. But cutoff value needs to be explored. In previous studies, ${ }^{9-12}$ 5-DS of scores of 1-3 were defined as negative scans, and scores of 4-5 were considered to be a positive result.

\section{Statistical Analysis}

PFS was described by median and range. Qualitative data were described by proportion and comparisons of qualitative data were performed by Chi-square test. Distribution of PFS was estimated by the Kaplan-Meier method, and comparisons of PFS were analyzed by log-rank test. Univariate and multivariate Cox's proportional-hazard model was used to estimate the hazard ratio and confidence interval of clinical prognostic factors. Data were analyzed using SPSS software (IBM Corp, Version 20.0, USA).

\section{Results \\ Description Of Patient Characteristics}

Patient demographics, clinical characteristics and 2-year progression status are displayed in Tables 1 and 2 .

As shown in Table 1, 8 of 59 patients (12 undergoing I-PET after 3 and 47 after 4 cycles, respectively) showed disease progression or recurrence. Of these 59 patients, 31 patients $(52.5 \%)$ were in advanced-stage disease (III/IV). Patients with high/medium-high IPI status accounted for $35.6 \%$. The proportion of high-risk patients in this study with 5-DS of 4-5 was $13.6 \%$ after I-PET measurement. According to the log-rank test, there was a statistical difference in PFS for IPI. There was no statistical difference in PFS when age, gender, and stage were taken into 
Table I Characteristics And 2-Year PFS Of 59 Patients Assessed By Interim PET/CT Scan After 3 Or 4 Cycles Of Therapy

\begin{tabular}{|l|l|l|l|l|l|}
\hline Variable & Value & Progression/n & 2-Year PFS (\%) & $\mathbf{X}^{\mathbf{2}}$ & $\mathbf{P}$ \\
\hline Age (year) & $>60$ & $4 / 25$ & 79.0 & 0.056 \\
& $\leq 60$ & $6 / 34$ & 79.8 & 0.813 \\
\hline Gender & Female & $4 / 34$ & 83.9 & 1.193 \\
& Male & $6 / 25$ & 73.1 & 0.275 \\
\hline IPI & High (IPI=3,4,5) & $7 / 21$ & 58.6 & 5.161 & 0.023 \\
\hline Stage & Low (IPI=0,I,2) & $3 / 38$ & 93.9 & 2.161 \\
\hline I-PET & High (Stage=3,4) & $8 / 31$ & 72.8 & 0.142 \\
\hline Stime (months) & Low (Stage=I,2) & $2 / 28$ & 91.5 & 2.063 \\
\hline Progress or not & Positive (DS=4,5) & $3 / 8$ & 57.1 & 83.4 & 0.151 \\
\hline
\end{tabular}

Abbreviations: 2-year PFS, two-year progression-free survival rate; IPI, International Prognostic Index; I-PET, interim positron emission tomography/computed tomography; Stime, survival time.

Table 2 Characteristics And 2-Year PFS Of 52 Patients Assessed By PET/CT Scan After 5 Or 6 Cycles Of Therapy

\begin{tabular}{|l|l|l|l|l|l|}
\hline Variable & Value & Progression/n & 2-Year PFS (\%) & $\mathbf{X}^{\mathbf{2}}$ & $\mathbf{P}$ \\
\hline Age & $>60$ & $5 / 21$ & 79.5 & 0.119 \\
\hline Gender & $\leq 60$ & $6 / 31$ & 76.5 & 0.730 \\
& Male & $5 / 23$ & 78.2 & 0.059 \\
\hline IPI & Female & $6 / 29$ & 69.0 & 0.808 \\
\hline Stage & High (IPI=3,4,5) & $8 / 22$ & 49.1 & 6.598 & 0.010 \\
\hline E-PET & Low (IPI=0,I,2) & $3 / 30$ & 78.9 & 1.185 \\
\hline Stime (Months) & High (Stage=3,4) & $9 / 32$ & 70.3 & 0.178 \\
Progress or not & Low (Stage=I,2) & $2 / 20$ & 94.1 & 11.46 \\
\hline
\end{tabular}

Abbreviations: 2-year PFS, two-year progression-free survival rate; IPI, International Prognostic Index; I-PET, interim positron emission tomography/computed tomography; Stime, survival time; min, minimum; max, maximum.

account. Similar findings were identified for I-PET with 5DS of 4-5 $(P=0.151)$.

As shown in Table 2, 5 patients underwent E-PET after 5 cycles of treatment and 47 patients after 6 cycles. There were no same patients underwent I-PET and E-PET. Eleven of 52 patients experienced progression or recurrence. According to the log-rank test, there was no statistical difference in PFS when age, gender, and stage were accounted for. There was a statistical difference in PFS between high and low IPI $(P=0.01)$. The 2 -year PFS was statistically different $(P=0.001)$ between positive and negative E-PET scans. The 2-year PFS was 34.6\% in the positive group and $91.7 \%$ in the negative group, in which 5 $\mathrm{DS} \geq 3$ was considered to be positive.

\section{Predictive Values Of I-PET For The Prognosis Of DLBCL}

The patients with 5-DS of 4-5 denoted by DS (score 4) were classified as having positive scans and were commonly used in the previous studies. ${ }^{9-12}$ There was no significant difference in PFS between negative and positive I-PET scans by DS (score 4) (Log-rank test, $P=0.151$ ). 
Kaplan-Meier curves for all patients with 5-DS scores 1-2, score 3, and score 4-5 are displayed in Figure 1A. The PFS curves of all patients are displayed in two clustered prognostic groups. PFS was identical between those with 5-DS score 3 and those with scores of 4-5. However, patients with a score of 3 in I-PET showed inferior PFS compared with those with scores of 1-2.

Of 9 patients with 5-DS score 3 , there were 5 with low IPI values and 4 with high IPI values. Of the 5 low IPI patients, no patient experienced progression, with a 2-year PFS of $100.0 \%$, whereas 3 of the 4 patients with high IPI experienced progression, leading to a 2 -year PFS of $25.0 \%$, which is shown in Table 3. We further subdivided patients with 5DS score 3 as positive I-PET scan when IPI was high and negative I-PET scan when IPI was low. This group division criterion combined 5-DS and IPI, resulting in the I-PET negative group consisting of patients with 5-DS score 1-2 or 3 but low IPI, while the I-PET-positive group consisted of patients with 5 -DS score $4-5$ or 3 but high IPI. Under this group division (denoted by DS+IPI), there was a significant difference in PFS between I-PET-positive and I-PET-negative patients $(P=0.001)$, which is shown in Table 4 . The 2 year PFS of the positive and negative scan groups was $95.3 \%$ and $45.5 \%$, respectively $(\mathrm{HR}=6.36(95 \%$ CI $[1.79,22.60])$, derived by univariate Cox regression). Compared with DS (score 4), with the cutoff value at score 4 , the combination criteria of 5-DS and IPI were more sensitive in separating good from poor prognosis patients.

The predictive values of I-PET, according to different cutoff values, are summarized in Table 5. When 5-DS scores of 4-5 were classified as positive PET scans, denoted by DS (score 4), 8 positive cases (13.6\%) were detected among 59 patients. Among these positive cases, 37.5\% (3/8) experienced progression in 2 years. Negative predictive values of DS (score 4) were 88.2\% (45/51), and area under curve (AUC) was $0.62(0.40-0.84)$. For the combined index DS + IPI, the number of positive patients increased to 12 , and of these 12 patients, 6 experienced progression in 2 years. The positive predictive value of DS+IPI was 50.0\%. Among 47 patients with negative PET scans, only 3 experienced progression. In terms of predictive indices, sensitivity, the positive predictive values (PPV) and negative predictive values (NPV) of DS+IPI were all higher than DS (score 4) $(66.7 \%$ vs $33.3 \%, 50.0 \%$ vs $37.5 \%, 93.6 \%$ vs $88.2 \%$ ), whereas specificity was almost same as DS (score 4) (88.0\% vs $90.0 \%)$. Compared with DS (score 4), the predictive accuracy of DS + IPI was greatly improved with $\mathrm{AUC}=0.77$ (0.58-0.97).

Age, gender, IPI, stage, and DS+IPI were included in multivariate Cox regression models to evaluate significant risk factors for PFS for 59 patients, which are summarized in Table 6. As a result, only DS+IPI was found to be associated with PFS in multivariate analysis $(P=0.004)$.

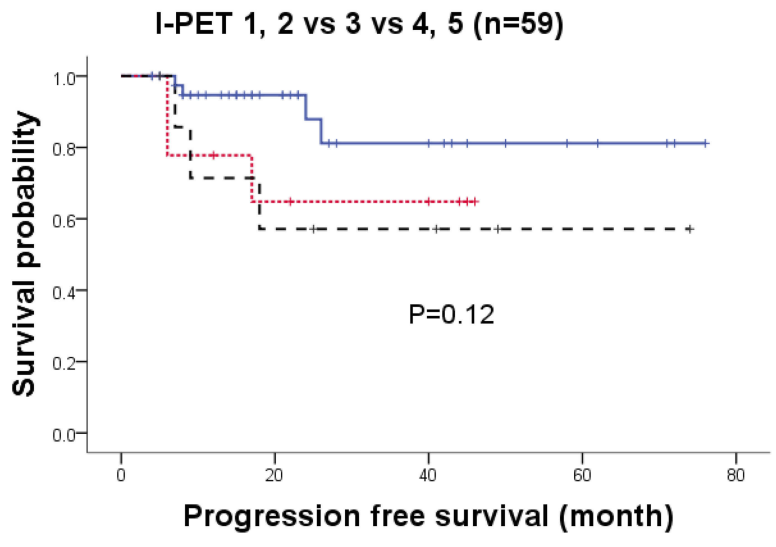

(a)

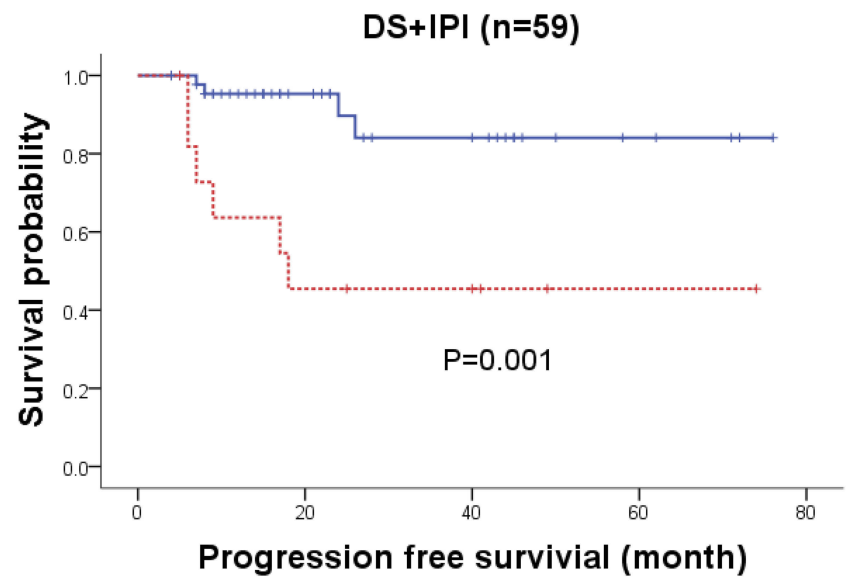

(b)

Figure I Kaplan-Meier curves for PFS of I-PET. (A) Kaplan-Meier curves for PFS of I-PET with cutoff at I-2 vs 3 vs $4-5$ for 59 patients treated by R-CHOP. The line represents 5-DS score I and 2, while the dashed line represents 5-DS score 3 and the dotted line represents 5 -DS score 4 and 5 . Log-rank test=4.25, $P=0.12, \mathrm{HR}=I .68$ (95\% $\mathrm{CI}[0.89,3.19])$. (B) Kaplan-Meier curves for PFS of patients stratified by the combination of I-PET and IPI. The line represents I-PET negative scan with 5-DS of I-2 or 5-DS score 3 but low IPI, while the dotted line represents PET/CT positive scan with 5-DS score $4-5$ or DS score 3 but high IPI. Log-rank test=I0.86, $P=0.00 I, \mathrm{HR}=6.36$ (95\% $\mathrm{Cl}$ $[1.79,22.56])$.

Abbreviations: PFS, progression-free survival; I-PET, interim PET/CT; HR, hazard ratio; CI, confidence interval; IPI, International Prognostic Index; 5-DS, Deauville 5-point scale. 
Table 3 Prognosis Of 9 Patients By Interim PET/CT Scan With 5-DS Of Score 3

\begin{tabular}{|l|l|l|l|l|}
\hline Variable & Value & Progression/n & 2-Year PFS (\%) & $\mathbf{P}$ \\
\hline IPI & $\begin{array}{l}\text { High } \\
(\mathrm{IPI}=3,4,5)\end{array}$ & $3 / 4$ & 25.0 & 0.023 \\
\hline & $\begin{array}{l}\text { Low } \\
(\mathrm{IPI}=0,1,2)\end{array}$ & $0 / 5$ & 100.0 & \\
\hline
\end{tabular}

Abbreviations: 2-year PFS, two-year progression-free survival rate; IPI, International Prognostic Index.

\section{Predictive Capacity Of E-PET For Prognosis Of DLBCL}

Fifty-two patients underwent E-PET after five or six cycles of therapies in our study. When 5-DS of 4-5 were classified as having positive scans, there was a significant difference in PFS between patients with negative E-PET scans and those with positive E-PET scans (Log-rank test, $P=0.001$, HR=6.10 (95\% CI [1.84-20.21]), derived by Cox regression). Kaplan-Meier curves for E-PET are shown in Figure 2. The predictive performance of E-PET for 2-year PFS is summarized in Table 7.

Age, gender, IPI, stage, and E-PET were included in multivariate Cox regression, which is summarized in Table 8. Only E-PET was found to be associated with PFS. A positive E-PET result had a higher risk of disease progression.

\section{Discussion}

Nowadays, 5-DS criteria for interpretation of response evaluation with $\mathrm{PET} / \mathrm{CT}$ have been widely adopted in DLBCL. ${ }^{9-12,16}$ It represents simple, reproducible criteria for I-PET interpretation. ${ }^{7}$ Barrington et $\mathrm{al}^{17}$ reported that validated 5-point London Criteria, which is the antecedent of the Deauville scoring system, were sufficiently robust to be used in a multicenter setting, both for the conservative (score 3 interpreted as "negative") and sensitive readings (score 3 interpreted as "positive"). Another visual criteria for interpretation of ${ }^{18} \mathrm{~F}$-FDG PET is the International Harmonization Project (IHP) which defines a positive FDG-PET scan as FDG uptake that is more intense than mediastinal blood pool activity in lesions larger than $2 \mathrm{~cm}$ and more than background in lesions smaller than $2 \mathrm{~cm} .{ }^{18}$ That is, the cutoff value of considering as positive result by IHP is lower than DS (score 4). Some patients with score 3 of 5-DS criteria, which uptake more than the mediastinal blood pool but less than or equal to the liver uptake, would be divided into positive scan according to IHP criteria.

A recent meta-analysis ${ }^{6}$ of 20 studies comprising 2255 patients was conducted to assess the predictive value of visually assessed I-PET. Of 20 studies, 5-DS were used in 12 studies and IHP criteria were adopted in four. Other visual criteria which were not based on consensus guidelines were used in the four remaining studies. Burggraaff et $\mathrm{al}^{6}$ reported that higher sensitivity values seen in the ROC analyses for both IHP and custom criteria vs 5-DS might be explained by the lower threshold of test positivity with IHP vs 5 -DS (using blood and liver pool activities as the reference tissues, respectively). To evaluate sensitivity and specificity comprehensively for 2-year PFS, we furthermore derived the Yoden Index (Yoden index $=$ sensitivity + specificity-1) of 19 available studies in Burggraaff et al. ${ }^{6}$ The Yoden index of three studies (except one study without

Table 4 Univariate Analysis For PFS By Different Cutoff Value Of I-PET Scan

\begin{tabular}{|l|l|l|l|l|l|}
\hline Variable & Value & Progression/n & 2-Year PFS(\%) & P & HR \\
\hline \multirow{2}{*}{ DS (score 4) } & Positive (DS=4,5) & $3 / 8$ & 57.1 & 0.151 & $2.59[0.67,10.06]$ \\
& Negative (DS=I,2,3) & $7 / 51$ & 83.4 & & \\
\hline \multirow{2}{*}{ DS+IPI } & Positive (DS=4,5 or DS=3 and IPI=positive) & $6 / 12$ & 45.5 & 0.001 & $6.36[1.79,22.60]$ \\
& Negative (DS=1,2 or DS=3 and IPI=negative) & $4 / 47$ & 95.3 & \\
\hline
\end{tabular}

Abbreviations: 2-year PFS, two-year progression-free survival rate; IPI, International Prognostic Index; DS (score 4), Deauville 5-point scale (5-DS), scores of 4-5 were classified as a positive scan; DS+IPI, Combination of 5-DS and IPI in the way of dividing patients with 5-DS score 3 by IPI.

Table 5 Predictive Accuracy Of 5-DS (Score 4) And DS+IPI For 2-Year PFS

\begin{tabular}{|l|l|l|l|l|l|}
\hline PET/CT & Sensitivity (\%) & Specificity (\%) & PPV (\%) & NPV (\%) & AUC (95\% CI) \\
\hline DS (score 4) & $33.3(3 / 9)$ & $90.0(45 / 52)$ & $37.5(3 / 8)$ & $88.2(45 / 51)$ & $0.62(0.40-0.84)$ \\
DS+IPI & $66.7(6 / 9)$ & $88.0(44 / 50)$ & $50.0(6 / 12)$ & $93.6(44 / 47)$ & $0.77(0.58-0.97)$ \\
\hline
\end{tabular}

Abbreviations: PPV, positive predictive value; NPV, negative predictive value; PFS, progression-free survival; DS (score 4 ) indicates DS $\geq 4$ as positive; DS+IPI, 5-DS of I to 2 as negative, 4 to 5 as positive, score 3 but high IPI as positive, score 3 but low IPI as negative; AUC, area under the curve. 
Table 6 Multivariate Analysis For PFS In Patients With I-PET

\begin{tabular}{|l|l|l|l|l|l|}
\hline Variables & B & Wald $\mathbf{X}^{2}$ & $\mathbf{P}$ & $\mathbf{H R}$ & $\mathbf{9 5 \%} \mathbf{C l}$ \\
\hline DS+IPI & 1.85 & 8.19 & 0.004 & 6.36 & {$[1.79,22.60]$} \\
\hline
\end{tabular}

Abbreviations: IPI, International Prognostic Index; DS+IPI, Combination of 5-DS and IPI in the way of dividing patients with 5-DS score 3 by IPI; HR, hazard ratio; $\mathrm{Cl}$, confidence interval.

reported sensitivity and specificity) with IHP criteria ranged from $28 \%$ to $42 \%$ and ranked 5th, 10th, and 13th of 19 studies from high to low according to the Yoden index. The 12 studies using 5-DS ranged from $13 \%$ to $58 \%$ in the Yoden index, indicating that the predictive accuracy of the IHP criteria was comparable with the Deauville system. The patients with 5-DS scores of 3 were those with uptake intensity between that of the mediastinum and the liver, which are considered to represent partial progression. According to the results of meta-analysis, ${ }^{6}$ if they were classified as positive, then sensitivity of I-PET for prognosis increased but specificity decreased. However, if they were classified as negative scans, then sensitivity decreased but specificity increased. This illustrates that patients with 5-DS scores of 3 are heterogeneous with differing prognoses. To subdivide patients with score 3 based on other predictive factors may improve the predictive accuracy.

In this study, for I-PET, there was no significant difference in PFS between I-PET positive and negative patients using the cutoff value of I-PET (5-DS score 4). The IPI is a wellestablished tool for the prediction of prognosis according to

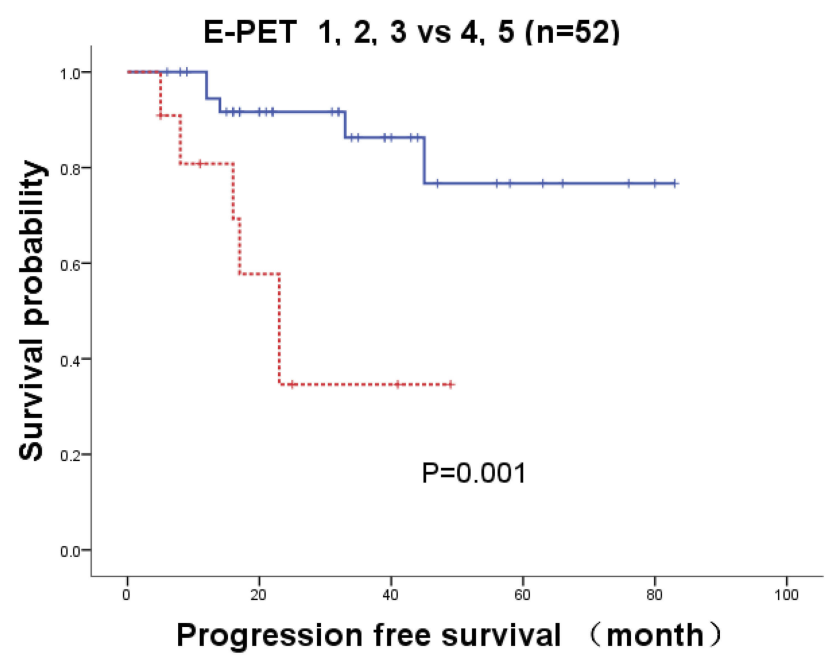

Figure 2 Kaplan-Meier curves for PFS of E-PET. Solid line represents negative E-PET scan with 5-DS score 1-3; dotted line represents positive E-PET scan with 5DS score 4-5. Log-rank test $=11.46, P=0.00 I$, HR=6.10 (95\% Cl[I.84-20.2I]). Abbreviations: PFS, progression-free survival; E-PET, patients underwent PET/ $\mathrm{CT}$ after five or six cycles of treatment; $\mathrm{HR}$, hazard ratio; $\mathrm{Cl}$, confidence interval. pretreatment characteristics. In this study, patients with 5-DS score 3 were subdivided into I-PET-positive when their IPI was high, and I-PET-negative when their IPI was low. Under this subgroup division, there was a significant difference in PFS between I-PET-positive and I-PET-negative patients $(P=0.001)$. Its sensitivity for 2-year PFS was higher than DS (score 4 ) (66.7\% vs 33.3\%), whereas its specificity was almost identical to DS (score 4$)(88.0 \%$ vs $90.0 \%)$. Furthermore, the PPV and NPV of this combination were both higher than DS (score 4) $(50.0 \%$ vs $37.5 \%, 93.6 \%$ vs $88.2 \%)$. Importantly, subdivision of patients with 5-DS score 3 by IPI improved the prediction accuracy.

In a previous study, Chow et $\mathrm{al}^{13}$ combined I-PET with IPI as a nomogram based on a bootstrapped Cox regression model. However, the cutoff value of this nomogram was not given, making it difficult for clinicians to discriminate between good- or poor-outcome patients. The clinical implications of the model were also hard for a clinician to understand. Nols et $\mathrm{a}^{14}$ divided patients into three groups: 1) double I-PET and IPI positive; 2) double I-PET and IPI negative; and 3) a group with only one positive index. Nols et al ${ }^{14}$ did not classify the group of patients for whom only one index was positive as either a positive group with poor prognosis or a negative group with good prognosis. Thus, it was still difficult for clinicians to decide on further treatment of these patients. Jiang et $\mathrm{al}^{19}$ divided patients into different risk groups using IPI and further subdivided them into risk groups using interim PET/CT. However, the authors encountered the same problem as Nols et al ${ }^{14}$ among patients with only one positive index (only IPI-positive or I-PET-positive); it was difficult for clinicians to discriminate good- or pooroutcome patients. Compared with other combinations of I-PET and IPI, dividing patients based on the cutoff value of 5-DS by IPI was easily accepted by clinicians and practical when considering further treatment options.

Instead of DS scores of 4-5 which are usually considered to be a positive result (denoted by DS (score 4)), Kim et al ${ }^{8}$ showed that a cutoff score of 5 by I-PET (denoted by DS (score 5)) had alternative clinical implications for identifying patients at significant risk. Changing the cutoff values of DS would result in increases in some predictive indices, but decreases in others. Kim et $\mathrm{al}^{8}$ found that compared with DS (score 4), DS (score 5) had higher specificity $(96.4 \%$ vs $74.8 \%)$, but sensitivity decreased by more than $10 \%(26.6 \%$ vs $38.5 \%$ ). That indicated that more than $10 \%$ of the pooroutcome patients could not be detected, which could confound further treatment decisions. Compared with DS (score 
Table 7 Predictive Accuracy Of E-PET For 2-Year PFS $(n=52)$

\begin{tabular}{|l|l|l|l|l|l|}
\hline Variable & Sensitivity (\%) & Specificity (\%) & PPV (\%) & NPV (\%) & AUC (95\% CI) \\
\hline E-PET & $66.7(6 / 9)$ & $88.4(38 / 43)$ & $54.5(6 / 11)$ & $92.7(38 / 4 I)$ & $0.78(0.58-0.87)$ \\
\hline
\end{tabular}

Abbreviations: E-PET, patients underwent PET/CT after five or six cycles of treatment. AUC, area under the curve; PPV, positive predictive value; NPV, negative predictive value; PFS, progression-free survival; AUC, area under the curve.

Table 8 Multivariate Analysis Of Prognosis For Patients With E-PET ( $n=52)$

\begin{tabular}{|l|l|l|l|l|l|}
\hline Variables & B & Wald $\mathbf{X}^{\mathbf{2}}$ & $\mathbf{P}$ & HR & 95\%C52I \\
\hline E-PET & 1.81 & 8.75 & 0.003 & 6.10 & $1.84-20.21$ \\
\hline
\end{tabular}

Abbreviations: E-PET, patients underwent PET/CT after five or six cycles of treatment; $\mathrm{HR}$, hazard ratio; $\mathrm{Cl}$, confidence interval.

4), we found that the predictive accuracy of DS (score 5) was not improved in our study, achieving slightly higher specificity $(96.0 \%$ vs $90.0 \%)$ at the cost of much lower sensitivity (11.1\% vs 33.3\%). However, the sensitivity of DS+IPI was much higher than DS (score 4) $(66.7 \%$ vs $33.3 \%)$, whereas the specificity was nearly the same $(88.0 \%$ vs $90.0 \%)$. Thus, IPI adds value to DS in I-PET, and DS+IPI was superior to changing the cutoff values of DS for detection of patients with good or poor prognosis.

Meignan et $\mathrm{al}^{7}$ reported the consensus guideline of 5-DS classification but did not define the cutoff value of 5-DS for classification of positive or negative scans. They reported that for 5-DS of scores 2-4, correction methods using the $\triangle$ SUVmax should be investigated. $\triangle \mathrm{SUVmax}^{20}$ and SUVmax-liver ${ }^{21}$ at I-PET have been reported to be a more sensitive method of separating good- from poor-outcome patients. However, it may be less practical because of the demanding conditions required for accurate and reproducible results. ${ }^{22}$ Further validation is needed to determine whether such semiquantitative factors add strength to 5-DS to stratify patients accurately.

Some limitations of our study are worth noting. This is a single-center, retrospective study with relatively small sample size. However, our data suggest that combination of 5-DS score with IPI can improve the predictive value of PET/CT in terms of patient prognosis. The combined criteria of I-PET and IPI should be refined in prospective trials and may help inform clinical decisions in treatment of DLBCL.

\section{Conclusion}

In conclusion, in the way of dividing the patients around the cutoff value of 5-DS with I-PET by IPI, we were able to discriminate between DLBCL patients with good or poor prognosis reliably. This approach was easily understood by clinicians and allowed them to decide on further treatment practically. Moreover, not only do this approach inform prognosis and further treatment, it provides a new way of combining I-PET criteria and other predictors to improve the predictive ability for the prognosis of DLBCL.

\section{Acknowledgment}

This research work was funded by National Science Foundation for Young Scholars of China (Grant No.8150 2891) and the National Natural Science Foundation of China (Grant No. 81872715).

\section{Disclosure}

The authors report no conflicts of interest in this work.

\section{References}

1. Mamot C, Klingbiel D, Hitz F, et al. Final results of a prospective evaluation of the predictive value of interim positron emission tomography in patients with diffuse large B-cell lymphoma treated with R-CHOP-14 (SAKK 38/07). J Clin Oncol. 2015;33(23):2523-2529. doi:10.1200/JCO.2014.58.9846

2. Adams HJ, Kwee TC. Prognostic value of interim FDG-PET in R-CHOP-treated diffuse large B-cell lymphoma: systematic review and meta-analysis. Crit Rev Oncol Hematol. 2016;106:55-63. doi:10. 1016/j.critrevonc.2016.07.003

3. Jiang M, Chen P, Ruan X, et al. Interim 18F-FDG PET/CT and BCL2 for predicting the prognosis of patients with diffuse large B-cell lymphoma in the rituximab era. Nucl Med Commun. 2018;39 (2):147-153. doi:10.1097/MNM.0000000000000784

4. Yuan L, Kreissl MC, Su L, et al. Prognostic analysis of interim 18 F-FDG PET/CT in patients with diffuse large B cell lymphoma after one cycle versus two cycles of chemotherapy. Eur J Nucl Med Mol Imaging. 2019;46(2):478-488. doi:10.1007/s00259-018-4198-6

5. Oñate-Ocaña LF, Cortés V, Castillo-Llanos R, et al. Metabolic tumor volume changes assessed by interval 18 fluorodeoxyglucose positron emission tomography-computed tomography for the prediction of complete response and survival in patients with diffuse large B-cell lymphoma. Oncol Lett. 2018;16(2):1411-1418. doi:10.3892/ol.2018.8817

6. Burggraaff $\mathrm{CN}$, de Jong A, Hoekstra OS, et al. Predictive value of interim positron emission tomography in diffuse large B-cell lymphoma: a systematic review and meta-analysis. Eur J Nucl Med Mol Imaging. 2019;46(1):65-79. doi:10.1007/s00259-018-4103-3

7. Meignan M, Gallamini A, Haioun C. Report on the First International Workshop on Interim-PET-scan in lymphoma. Leuk Lymphoma. 2009;50(8):1257-1260. doi:10.1080/10428190903040048

8. Kim J, Song YS, Lee JS, Lee WW, Kim SE. Risk stratification of diffuse large B-cell lymphoma with interim PET-CT based on different cutoff Deauville scores. Leuk Lymphoma. 2018;59(2):340-347. doi:10.1080/10428194.2017.1339877 
9. Safar V, Dupuis J, Itti E, et al. Interim [18F]fluorodeoxyglucose positron emission tomography scan in diffuse large B-cell lymphoma treated with anthracycline-based chemotherapy plus rituximab. J Clin Oncol. 2012;30(2):184-190. doi:10.1200/JCO.2011.38.2648

10. Yang DH, Ahn JS, Byun BH, et al. Interim PET/CT-based prognostic model for the treatment of diffuse large B cell lymphoma in the postrituximab era. Ann Hematol. 2013;92(4):471-479. doi:10.1007/s00277012-1640-X

11. Yang DH, Min JJ, Song HC, et al. Prognostic significance of interim (1)(8)F-FDG PET/CT after three or four cycles of R-CHOP chemotherapy in the treatment of diffuse large B-cell lymphoma. Eur J Cancer. 2011;47(9):1312-1318. doi:10.1016/j.ejca.2010.12.027

12. Pregno P, Chiappella A, Bello $\mathrm{M}$, et al. Interim 18-FDG-PET/CT failed to predict the outcome in diffuse large B-cell lymphoma patients treated at the diagnosis with rituximab-CHOP. Blood. 2012;119(9):2066-2073. doi:10.1182/blood-2011-06-359943

13. Chow A, Phillips M, Siew T, et al. Prognostic nomogram for diffuse large B-cell lymphoma incorporating the International Prognostic Index with interim-positron emission tomography findings. Intern Med J. 2013;43(8):932-939. doi:10.1111/imj.12194

14. Nols N, Mounier N, Bouazza S, et al. Quantitative and qualitative analysis of metabolic response at interim positron emission tomography scan combined with International Prognostic Index is highly predictive of outcome in diffuse large B-cell lymphoma. Leuk Lymphoma. 2014;55 (4):773-780. doi:10.3109/10428194.2013.831848

15. Zelenetz AD, Gordon LI, Wierda WG, et al. Diffuse large B-cell lymphoma version 1.2016. J National Compr Cancer Network. 2016;14(2):196-231. doi:10.6004/jnccn.2016.0023

16. Cheson BD, Fisher RI, Barrington SF, et al. Recommendations for initial evaluation, staging, and response assessment of Hodgkin and non-Hodgkin lymphoma: the Lugano classification. J Clin Oncol. 2014;32(27):3059-3068. doi:10.1200/JCO.2013.54.8800
17. Barrington SF, Qian W, Somer EJ, et al. Concordance between four European centres of PET reporting criteria designed for use in multicentre trials in Hodgkin lymphoma. Eur J Nucl Med Mol Imaging. 2010;37(10):1824-1833. doi:10.1007/s00259-010-1490-5

18. Juweid ME, Stroobants S, Hoekstra OS, et al. Use of positron emission tomography for response assessment of lymphoma: consensus of the imaging subcommittee of international harmonization project in lymphoma. J Clin Oncol. 2007;25(5):571-578. doi:10.1200/JCO.2006.08. 2305

19. Jiang M, Chen P, Ruan X, et al. Interim 18F-FDG PET/CT improves the prognostic value of S-IPI, R-IPI and NCCN-IPI in patients with diffuse large B-cell lymphoma. Oncol Lett. 2017;14(6):6715-6723. doi:10.3892/ol.2017.7093

20. Itti E, Meignan M, Berriolo-Riedinger A, et al. An international confirmatory study of the prognostic value of early PET/CT in diffuse large B-cell lymphoma: comparison between Deauville criteria and DeltaSUVmax. Eur J Nucl Med Mol Imaging. 2013;40(9):1312-1320. doi:10.1007/s00259-013-2435-6

21. Zhang Y, Fan Y, Ying Z, et al. Can the SUVmax-liver-based interpretation improve prognostic accuracy of interim and posttreatment 18F-FDG PET/CT in patients with diffuse large B-cell lymphoma? Leuk Lymphoma. 2018;59(3):660-669. doi:10.1080/10428194.2017. 1357171

22. Carr R, Fanti S, Paez D, et al. Prospective international cohort study demonstrates inability of interim PET to predict treatment failure in diffuse large B-cell lymphoma. J Nucl Med. 2014;55(12):1936-1944. doi:10.2967/jnumed.114.145326

\section{Publish your work in this journal}

Cancer Management and Research is an international, peer-reviewed open access journal focusing on cancer research and the optimal use of preventative and integrated treatment interventions to achieve improved outcomes, enhanced survival and quality of life for the cancer patient.
The manuscript management system is completely online and includes a very quick and fair peer-review system, which is all easy to use. Visit http://www.dovepress.com/testimonials.php to read real quotes from published authors. 